\title{
Evaluación de la red de distribución: conexión del vehículo eléctrico híbrido enchufable
}

\section{Evaluation of the power distribution network: plug-in hybrid electric vehicle connected}

\author{
Jose Galarza-Linares ${ }^{1}$, David Condezo-Hurtado², \\ Bartolome Saenz-Loayza ${ }^{3}$, David Huarac-Rojas ${ }^{4}$
}

Fecha de recepción: 17 de setiembre de 2020

Fecha de aprobación: 27 de enero de 2021

Galarza-Linares, J; Condezo-Hurtado, D; Saenz-Loayza, B; Huarac-Rojas, D. Evaluación de la red de distribución: conexión del vehículo eléctrico híbrido enchufable. Tecnología en Marcha. Vol. 34-4 Octubre-Diciembre 2021. Pág 50-62.

doi) https://doi.org/10.18845/tm.v34i4.5384

1 Universidad Nacional del Centro del Perú. Perú. Correo electrónico: jgalarza@uncp.edu.pe (D) https://orcid.org/0000-0001-5569-6541

2 Universidad Nacional del Centro del Perú. Perú. Correo electrónico: dcondezo@uncp.edu.pe (D) https://orcid.org/0000-0002-1658-910X

3 Universidad Nacional del Centro del Perú. Perú. Correo electrónico: bsaenz@uncp.edu.pe (D) https://orcid.org/0000-0002-8664-8574

4 Universidad Nacional del Centro del Perú. Perú. Correo electrónico: dhuarac@uncp.edu.pe (i) https://orcid.org/0000-0003-1569-4917 


\title{
Palabras clave
}

Caída de tensión; demanda eléctrica; operación del sistema eléctrico; pérdidas de energía eléctrica; sistema eléctrico de prueba; vehículo eléctrico híbrido enchufable.

\section{Resumen}

La evaluación de nuevas tecnologías en el sistema eléctrico es pertinente para la planificación y operación de la red eléctrica, esto permite brindar un servicio eléctrico de calidad a los usuarios finales y preservar la operación del sistema eléctrico. La presente investigación analiza la integración de los vehículos eléctricos híbridos enchufables (VEHE) en la red eléctrica de distribución, el sistema eléctrico Europeo de Baja Tensión de 906 barras del IEEE es usado como sistema de prueba, considerando el incremento de la demanda eléctrica en función de la operación, horario de carga, tecnología y autonomía de esta nueva tecnología de movilidad. A través de una metodología analítico-no experimental, los siguientes factores fueron analizados: variación de tensión, pérdidas de energía eléctrica e incremento de la demanda en el mediano y largo plazo. Los resultados obtenidos muestran que para niveles de irrupción de esta nueva tecnología es necesario repotenciar la red eléctrica actual para preservar las condiciones de operación estables del sistema eléctrico y cumplir con la normativa del sector eléctrico, los resultados muestran que la conexión masiva y sin control tiene una gran repercusión en la operación de la red eléctrica.

\section{Keywords}

Voltage drop; power demand; power system operation; power loss; power system test; plug-in hybrid electric vehicle.

\begin{abstract}
The evaluation of new technologies in the electrical system is relevant for the planning and operation of the electrical network, this allows to provide a quality electrical service to the final users and to preserve the operation of the electrical system. The present research analyzes the integration of plug-in hybrid electric vehicles (EVs) in the electric distribution network. The European low voltage 906 busbar electric system of the IEEE is used as a test system, considering the increase of the electric demand depending on the operation, charging time, technology and autonomy of this new mobility technology. Through an analytical-non-experimental methodology, the following factors were analyzed: voltage variation, electrical energy losses and increase in demand in the medium and long term. The results obtained show that for levels of irruption of this new technology it is necessary to repower the current electrical network to preserve the stable operating conditions of the electrical system and to comply with the regulations of the electrical sector, the results show that the massive and uncontrolled connection has a great impact on the operation of the electrical network.
\end{abstract}

\section{Introducción}

Para la introducción de los vehículos eléctricos (VE) en la sociedad, es necesario el despliegue de ciertas estrategias: equipos de carga para VE, descarbonización en la producción de electricidad y la integración de los VE a la red eléctrica [1]. La tecnología del vehículo eléctrico híbrido enchufable (VEHE) con el uso del motor eléctrico para su propulsión, representa una tecnología más eficiente respecto al vehículo eléctrico híbrido convencional con motor de combustión [2]. El comportamiento de las personas en el uso de VEHE y las características 
según el tipo de VEHE, son determinantes para el estudio del impacto de la conexión de esta tecnología en la red eléctrica [3], este tipo de VE necesita una gran cantidad de energía para la carga de baterías, esta demanda de energía adicional podría tener impactos negativos en la red eléctrica [4].

El estudio de la planificación del sistema eléctrico de distribución con el ingreso de VEHE debe considerar los siguientes indicadores: pérdidas eléctricas y caída de tensión; por parte del VEHE es necesario conocer la capacidad de batería, nivel de carga, autonomía eléctrica, horario de carga entre otros factores [3]. En [5] se estudió el análisis del flujo de carga a través del control de tensión dependiente para obtener los indicadores mencionados en [3]. La conexión de los VEHE en forma descoordinada pueda afectar la operación estable del sistema, su confiabilidad y seguridad; las estrategias inteligentes en función de la tecnología de carga contribuyen a la seguridad de la red eléctrica y preservan las condiciones de operación económica estable [6]. En [7] se considera a los VEHE agentes energéticos en el sistema eléctrico, para la conexión de esta tecnología a la red eléctrica se analizó el tiempo de conexión a la red, la ubicación de centro de carga y el nivel de batería; a través de una simulación del sistema eléctrico se analizó esquemas de gestión inteligente de energía para aliviar la congestión en el sistema eléctrico debido a la conexión de esta tecnología en la red eléctrica. Los VEHE y las demás tecnologías de VE son evaluados en [8] a través de un marco de referencia que las integra en la red eléctrica a través de enfoques analíticos, deterministas y estocásticos.

El presente trabajo se enmarca en las disposiciones de eficiencia energética que viene adoptando Perú, específicamente en la inclusión de VE en el parque automotor. En este contexto las redes eléctricas de distribución abastecerán de energía a estos vehículos, en consecuencia, estas redes estarán sometidas a un nuevo "stress eléctrico" sobre su funcionamiento actual. La presente investigación evalúa la carga del VE desde la red eléctrica, conocida como G2V (grid to vehicle) [1]. El objetivo es analizar el comportamiento de la red eléctrica en Baja Tensión ante un panorama de conexiones masiva de VEHE, el análisis de las pérdidas eléctricas, caída de tensión y la variación de la demanda, concluyen que el sistema eléctrico debe ser repotenciado para conservar su funcionamiento en los límites normales de operación. El carácter predictivo del proyecto contempla situar a las redes de distribución peruanas dentro de un funcionamiento con los niveles de confiabilidad y fiabilidad establecidos por la normativa del Sub Sector de Electricidad Peruano así mismo afrontar los nuevos escenarios a los que serán sometidos las redes eléctricas sin perjudicar la calidad del servicio eléctrico para los usuarios y la operación del sistema eléctrico.

\section{Materiales y métodos}

\section{Materiales}

\section{Sistema Eléctrico de Prueba del IEEE (SEP-IEEE):}

EI SEP-IEEE utilizado en la presente investigación es un modelo matemático de un sistema de distribución en Baja Tensión (BT) Europeo, las especificaciones técnicas, así como las condiciones de operación fueron establecidas por IEEE. Este sistema es denominado IEEE European Low Voltage Test Feeder que pertenece al grupo IEEE PES Distribution System Analysis Subcommittee's Distribution Test Feeder Working Group [10]. A continuación, se menciona las características importantes del SEP-IEEE:

Sistema de Distribucíon radial en BT de 906 barras, tensión del sistema 416 V a 50 Hz, típica del sistema BT Europeo. 
El alimentador del SEP-IEEE está conectado a la red MT a través de un transformador $(11 / 0.416 \mathrm{kV})$.

El sistema presenta 55 cargas, cada una con un perfil de carga diferente, la información del consumo energético está en series de tiempo en intervalos de 1 minuto durante las $24 \mathrm{~h}$.

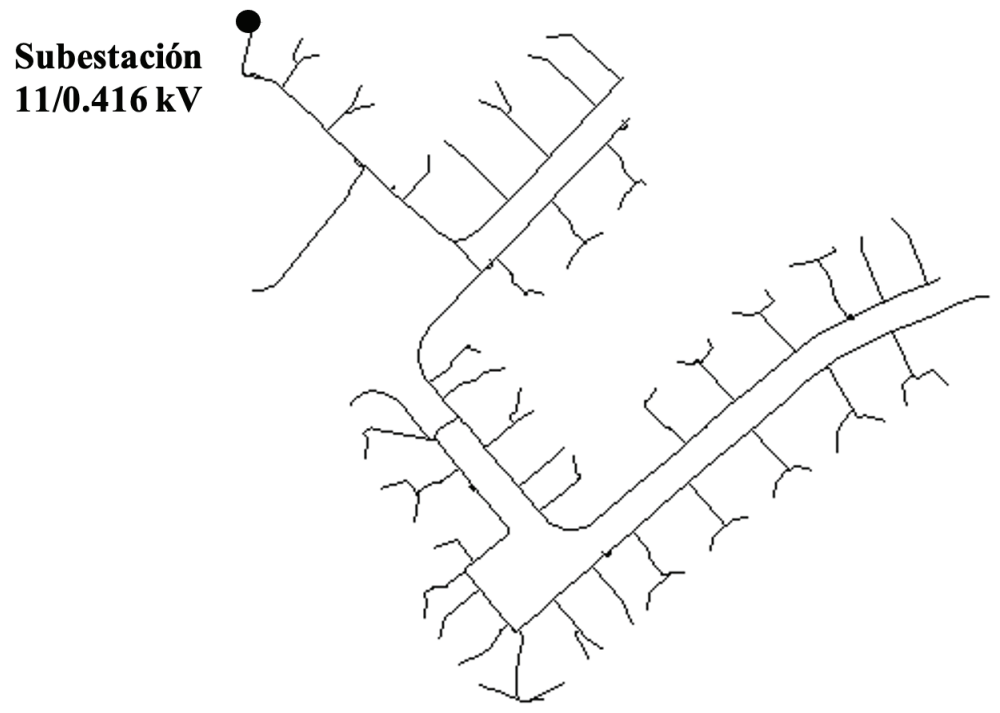

Figura 1. Sistema eléctrico IEEE de prueba (906 barras). Fuente: [10]

\section{Entorno de Simulación:}

Para la presente investigación, se consideró el modelo de red desarrollado por la empresa MathWorks [11], donde considera las 906 barras del sistema en BT. El Cuadro 1 muestra las especificaciones del software Matlab/Simulink y las herramientas utilizadas en la presente investigación, los cálculos eléctricos se realizaron a través de un script en Matlab.

Cuadro 1. Parámetros del entorno de simulación.

\begin{tabular}{|c|c|}
\hline Parámetro & Descripción \\
\hline \multirow{2}{*}{ Herramientas } & Matlab Versión 9.3 \\
\cline { 2 - 2 } & Simulink Versión 9.0 \\
\cline { 2 - 2 } & Simscape Power Systems Versión 6.8 \\
\hline Versión Matlab/Simulink & 9.3 .0 .948333 (R2017b) \\
\hline Tipo de Simulación & Fasorial (Función acelerada activada) \\
\hline Tiempo de Simulación (s) & $86400(24 \mathrm{~h})$ \\
\hline Paso de tiempo - Ts (s) & 1 \\
\hline Método de Solución (Solver) & Automático - Paso fijo (Ts) - ode 14x \\
\hline
\end{tabular}

Consideraciones de operación del VEHE:

Esta sección define los criterios para estimar el comportamiento de los usuarios:

- Los usuarios que tiene vehículo propio son utilizados para desplazamiento propio y solo para fines de transporte de personas (la capacidad de carga y remolque no es considerada). 
- Los usuarios que utilizan automóviles son personas que corresponden al sector residencial, que generalmente recorren en promedio 20 - 30km diarios en ida y vuelta desde su hogar a su centro de trabajo.

- Los usuarios en promedio tienen el horario de retorno hacia sus hogares en el intervalo de tiempo de 6 pm-8 pm.

- Los usuarios en promedio tienen un solo vehículo el cual utilizan los días de semana y los fines de semana. Se ha considerado la evaluación de un día de la semana cualquiera.

- La incursión progresiva hasta el 50\% de VEHE corresponde al 2060 en adelante.

\section{Horario de carga VEHE:}

El horario de llegada a una estación coincide con el horario de culminación de un viaje, es el horario de inicio del proceso de carga del VEHE. La figura 2 muestra la distribución del horario de llegada al hogar, indicando el porcentaje de VEHE que comienzan con el periodo de carga.

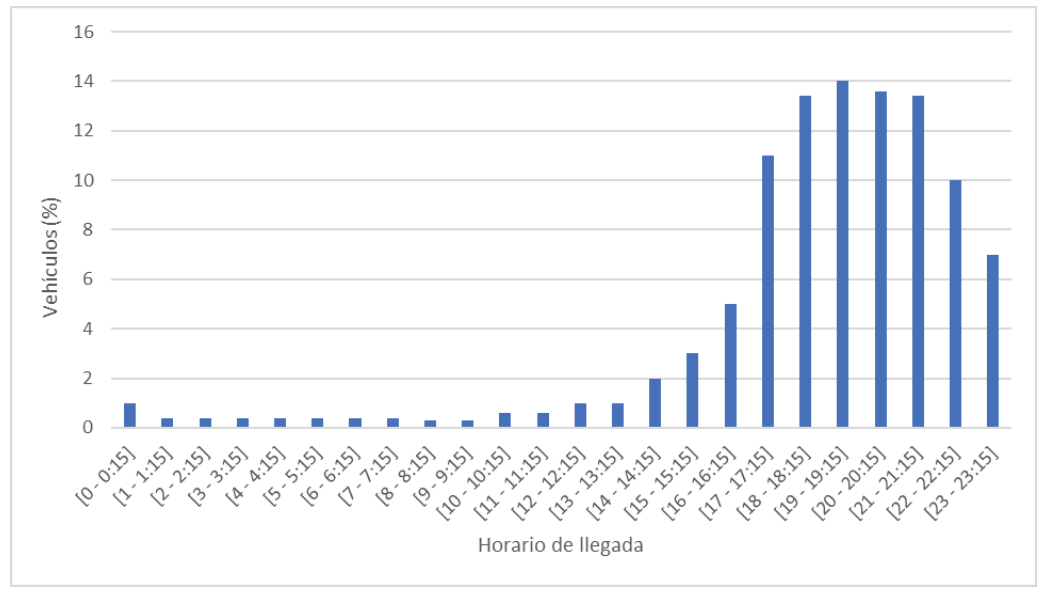

Figura 2. Distribución de la conexión de VEHE según horario de carga.

\section{Tipos de VEHE:}

En esta investigación se consideran los tipos de automóviles más comunes (Sedan y SUV). El Laboratorio Nacional del Noroeste del Pacífico realizó la estimación de la energía eléctrica consumida por kilómetro (EECK) para diferentes tipos de VEHE [12], considerando este criterio se elaboró el cuadro 2 donde se muestra 4 tipos de vehículos y sus valores EECK.

Cuadro 2. Características del VEHE según el tipo de vehículo.

\begin{tabular}{|c|c|c|c|c|}
\hline $\begin{array}{c}\text { Tipo de } \\
\text { Vehículo }\end{array}$ & $\begin{array}{c}\text { Sedan } \\
\text { compacto }\end{array}$ & $\begin{array}{c}\text { Sedan } \\
\text { mediano }\end{array}$ & SUV mediano & SUV grande \\
\hline Cantidad (\%) & 10 & 40 & 45 & 5 \\
\hline Autonomía & VEHE40 & VEHE50 & VEHE60 & VEHE60 \\
\hline EECK $(\mathrm{kW} / \mathrm{km})$ & 0.26 & 0.3 & 0.38 & 0.46 \\
\hline
\end{tabular}




\section{Autonomía del VEHE:}

Este criterio está en relación con las marcas comerciales que al 2020 están disponibles en el mercado internacional. La nomenclatura VEHEX establece los "X" kilómetros de autonomía eléctrica máxima del vehículo. El Cuadro 2 establece valores de autonomía eléctrica para VEHEE comerciales, los valores de autonomía eléctrica son los establecidos por el fabricante: sedán compacto (Mini Countryman Cooper S E All4), sedán mediano (Hyundai Ioniq PHEV), SUV mediano (Mitsubishi Outlander PHEV), SUV grande (Volvo XC90 T8 Twin Engine).

\section{Incremento de VEHE en el parque automor:}

Partiendo del parque automotor actual, la estimación del nivel de incursión de los VEHE para el mediano y largo plazo se muestra en la figura 3. En un escenario optimista, se considera un posicionamiento del 50\% de esta nueva tecnología desde el 2060 en adelante. En relación con el SEP-IEEE, el número de VEHE considerado para el 2060 son 175 unidades.

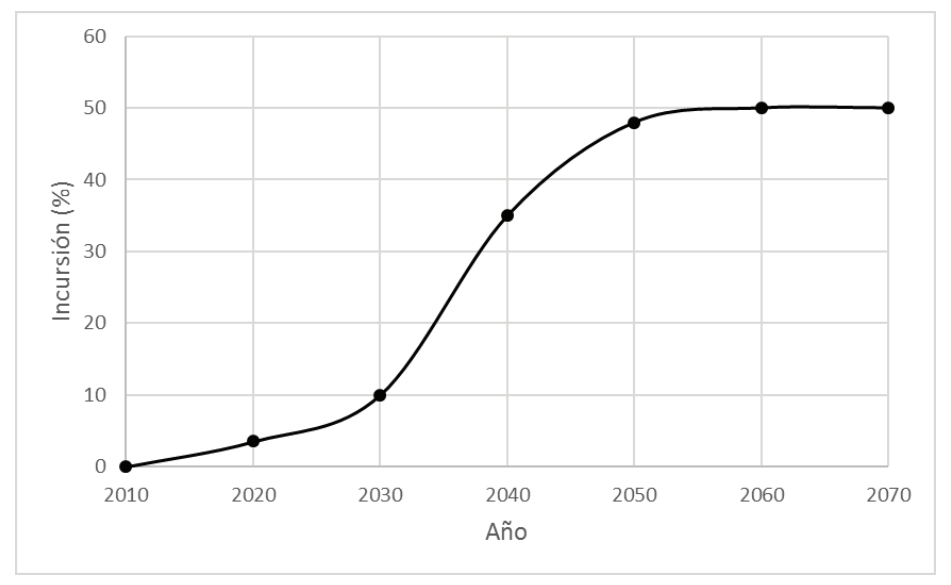

Figura 3. Incremento gradual de los VEHE en el parque automotor.

\section{Perfil de carga diario:}

El sistema eléctrico de distribución de prueba establecido en [10], tiene un perfil de carga con un consumo masivo desde las primeras horas de la mañana. Bajo las consideraciones establecidas en la sección: Consideraciones de operación del VEHE, se eligió el intervalo de 18:00 - 23:00 horas correspondientes al horario de carga.

\section{Estimación de la demanda:}

Para la presente investigación, el año 2010 es considerado como año base para realizar las proyecciones de demanda. La información del sistema de distribución presentado en [10] es considerada como información para el año de referencia. El Departamento de Energía de U.S.A, establece el crecimiento promedio 1\% anual de la demanda, para esta investigación se consideró la metodología descrita en [9]. 


\section{Métodos}

\section{Solución del modelo del sistema:}

MathWorks ofrece una variedad de modos de solución para ecuaciones diferenciales algebraicas, la elección se alguna depende del tipo de circuito eléctrico a resolver. Para la presente investigación se optó por usar un solver automático de tiempo de simulación constante (Ts), en la configuración como automático, MathWorks asigna el solver ode14x para resolver las ecuaciones diferenciales algebraicas de paso fijo y estados continuos como se presenta en el SEP-IEEE. El solver ode14x, realiza una combinación del método de Newton y el proceso de extrapolación usando los valores actuales para calcular el modelo de estado en el siguiente paso de tiempo [13].

\section{Incremento de la demanda por VEHE:}

La información suministrada por [10] presenta un perfil de carga por cada minuto para un día (24h), este consumo de demanda es el que será evaluado según la incursión de VEHE y según el crecimiento de la demanda. A continuación, se describe el procedimiento utilizado para evaluar el incremento del consumo de la demanda eléctrica.

- El año 2010 es tomado como referencia para el cálculo de proyección de la demanda, según [9] se procedió a incrementar la demanda en función del año de evaluación.

- Para el año de análisis se eligió el nivel de incursión de los VEHE, desde un nivel nulo hasta un 50\% en el 2060.

- Cada punto de carga corresponde al consumo de un conjunto de familias, el incremento de la demanda está en función a la cantidad, tipo y EECK del VEHE.

- Para los 55 puntos de carga en el sistema, se ha considerado el tipo de carga 0.2C durante 5 horas, el intervalo de tiempo seleccionado es de 18:00 h a 23:00 h. En forma aleatoria se realiza la distribución de VEHE en los puntos de carga, el nivel de energía que necesitan los VEHE es considerado igual a la capacidad de la batería.

\section{Pérdidas en el sistema eléctrico:}

Las redes de distribución están modeladas por una impedancia, a través de elementos pasivos: resistencias e inductancias. El procedimiento para el cálculo de pérdidas está basado en el consumo de potencia por la resistencia en los diferentes ramales de la red, las pérdidas totales están expresadas según (1).

$$
P_{t}=\sum_{i=1}^{n}\left(I_{a b c(i)}\right)^{2} \cdot R
$$

Donde el valor de $I_{a b c(i)}$ es el valor de la corriente que se inyecta a la carga ubicada en la barra $i$. El valor $R$ es la resistencia total del ramal por donde circula dicha corriente. La sumatoria de las pérdidas individuales de las $n$ ramas corresponde a las pérdidas totales del sistema de distribución.

\section{Desviación del nivel de tensión nominal:}

El cálculo de la desviación de tensión está en función de los valores obtenidos luego de resolver el circuito eléctrico a través de Matlab/Simulink, considerando cada una de las barras en el sistema eléctrico, se obtiene la desviación porcentual de tensión según (2). 


$$
\% V=\frac{V_{c}-V_{r}}{V_{r}}
$$

Donde $V_{c}$ es la tensión en el punto de carga, y $V_{r}$ es la tensión de referencia (416 V).

\section{Indicador de curva de demanda:}

El diagrama de carga total muestra la sumatoria de potencias de todas las cargas individuales en un intervalo de tiempo de 24h. El factor peak to average ratio (PAR), muestra en general la simetría respecto de la curva de demanda respecto a la potencia promedio, este indicador esta expresado según (3).

$$
P A R=\frac{P_{M A X}}{P_{P R O M}}
$$

Donde $P_{M A X}$ corresponde a la potencia máxima en el diagrama de carga y $P_{P R O M}$ esta referido a la potencia promedio.

\section{Resultados}

Esta sección muestra los casos evaluados para el análisis del comportamiento del SEP-IEEE. Este sistema eléctrico es evaluado convenientemente teniendo presente el nivel de incremento de carga en el mediano y largo plazo, y el incremento de VEHE en el parque automotor. Luego de realizar la simulación en el entorno de Matlab/Simulink los datos obtenidos de tensiones y corrientes son utilizados para realizar los siguientes cálculos: Demanda total en kW, Variación de la Tensión en \% por fase, Pérdidas totales en kW, Demanda máxima, Demanda promedio y el PAR.

\section{Caso I:}

El objetivo del presente caso es evaluar el comportamiento de la red eléctrica de distribución en condiciones normales de operación. Los resultados de P, Q e V suministrados en [10], son usados para validar los resultados del caso base. La información validada permitió implementar el SEP-IEEE con los criterios establecidos en la Sección: Incremento de la Demanda por VEHE. La curva de demanda total mostrada en la figura 4 presenta el pico de demanda (57kW) a las 9:40 am. con relación a esta información el PAR es 2.84. Los resultados, según la demanda de energía, permite concluir que las personas realizan actividades que consumen energía durante todo el día, el factor PAR hace referencia a un perfil de carga con picos de demanda que están muy por encima del valor promedio de la demanda. 


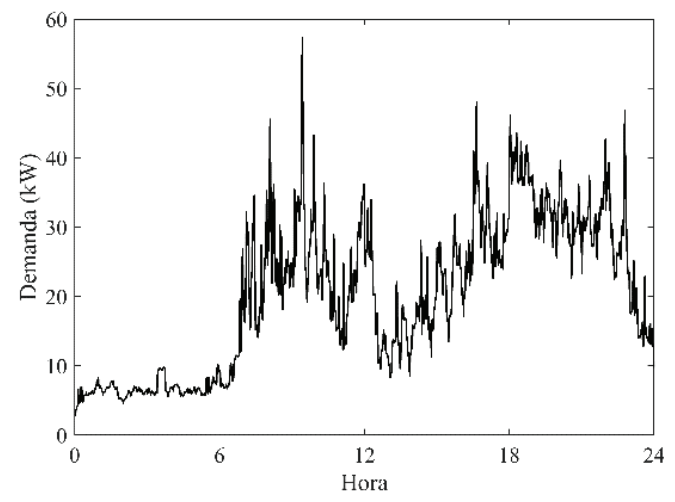

Figura 4. Caso I - Demanda total del sistema.

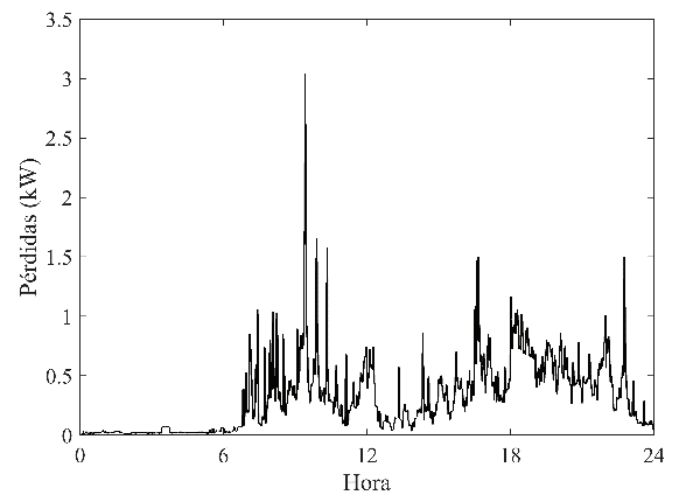

Figura 5. Caso I - Pérdidas totales en el sistema.

La figura 5 expresa las pérdidas totales del sistema en kW. Se registra un valor máximo de 3kW a las 9:40 horas pertenecientes al pico de demanda, las pérdidas totales representan el $5.2 \%$ del consumo en el horario mencionado. La figura 6 muestra la variación de tensión en la barra 32 para las tres fases, se presenta una caída de tensión máxima de 1.3\% en la fase B. La desviación de tensión en las 55 barras se muestra en la figura 7, el desbalance del sistema permite apreciar una mayor cargabilidad en la fase B, la fase C presenta la menor variación de tensión, cercana a 2\%.

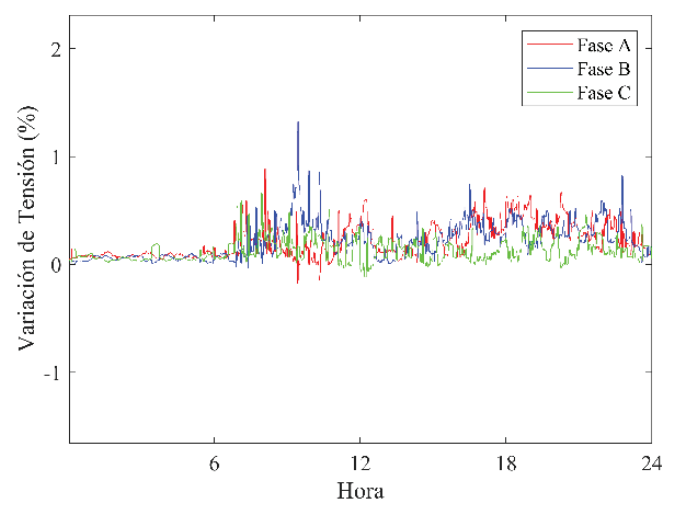

Figura 6. Caso I - Desviación de tensión barra 32.

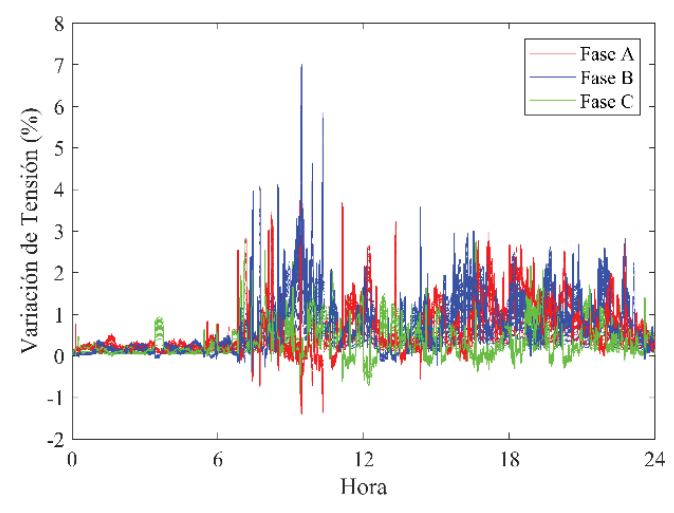

Figura 7. Caso I - Desviación de tensión en las 55 barras.

\section{Caso II:}

Para este caso se evalúa el nivel de incursión del 35\% de los VEHE correspondiente al 2040 y el incremento de la demanda correspondiente. La figura 8 muestra la comparación de la nueva demanda considerando la incursión de VEHE e incremento de la demanda. 


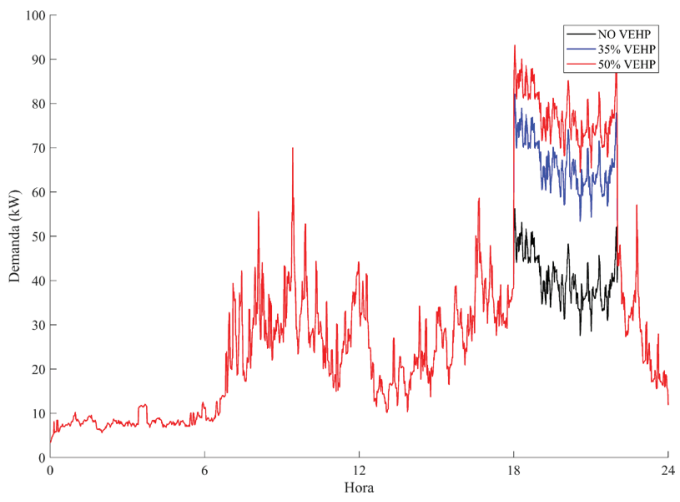

Figura 8. Caso II - Incremento del consumo energético con la incursión de VEHE.

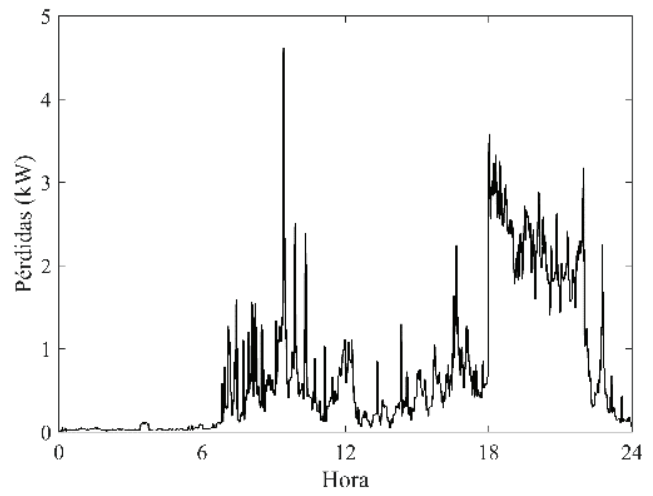

Figura 9. Caso II - Pérdidas totales en el sistema.

La máxima demanda es registrada a las 18:00 horas con $82 \mathrm{~kW}$, en consecuencia, el PAR=2.84. La figura 9 muestra las pérdidas en función del tiempo durante las 24h, a consecuencia del incremento del consumo energético, las pérdidas se incrementaron en el horario de carga de IOS VEHE. Al realizar la comparación entre la figura 5 y figura 9 , se determina que en el horario de inicio de carga del VEHE las pérdidas se incrementaron en 192\%, a las 9.42am se registra el mayor pico de pérdidas (4.6 kW). Se eligió la barra 34 para mostrar los nuevos incrementos de la variación de tensión, la figura 10 muestra la variación de la tensión en la barra 34, se presenta un máximo de $1.8 \%$ en la fase $B$.

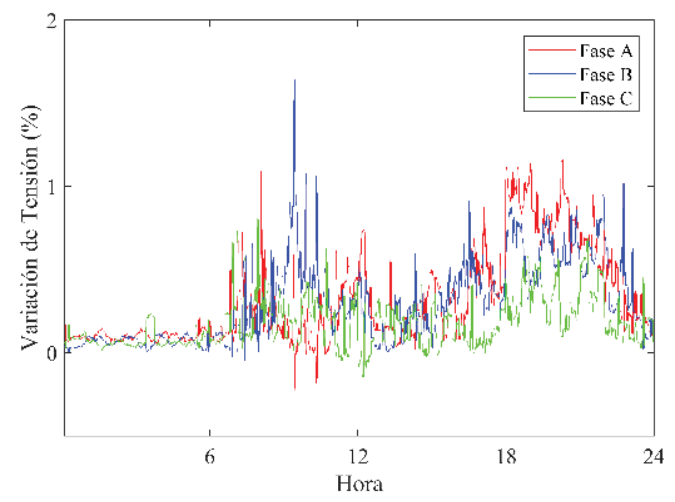

Figura 10. Caso II - Variación de tensión en la barra 34.

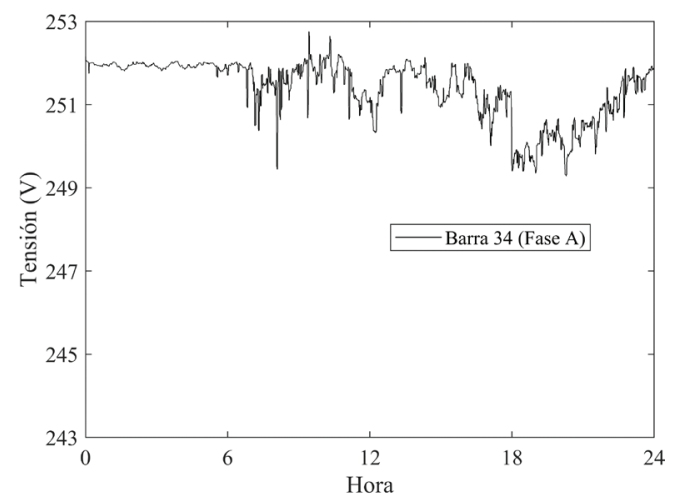

Figura 11. Caso II - Perfil de tensión en la barra 34.

La figura 11 muestra el nivel de tensión en la barra 34 (fase A), presentándose mayor desviación durante los periodos de carga del VEHE. En el horario de 18:00 - 23:00 horas se tiene la mayor disminución de la tensión debido al incremento del consumo de energía debido a la conexión de VEHE en la red de distribución. Se eligió la barra 906 (Carga 55), correspondiente a la barra más alejada desde el punto de transformación en 11/0.416 kV. La figura 12 muestra la variación de la tensión en la barra 906 correspondiente a las tres fases, al realiza la comparación con la figura 7 , se concluye que el nivel de caída de tensión se duplica en las tres fases. La figura 13 muestra el perfil de la tensión en la barra 906, se presenta una disminución sustancial de la tensión en el horario de conexión de VEHE. Con los resultados obtenidos se concluye que es necesario la instalación de equipos de compensación y repotenciación de 
la red de distribución. Los valores de caída de tensión están cercanos al 5\%, este es el límite que establece el Código Nacional de Electricidad de USA (NEC) y el Código Nacional de Electricidad Peruano (CNEP) para zonas urbanas.

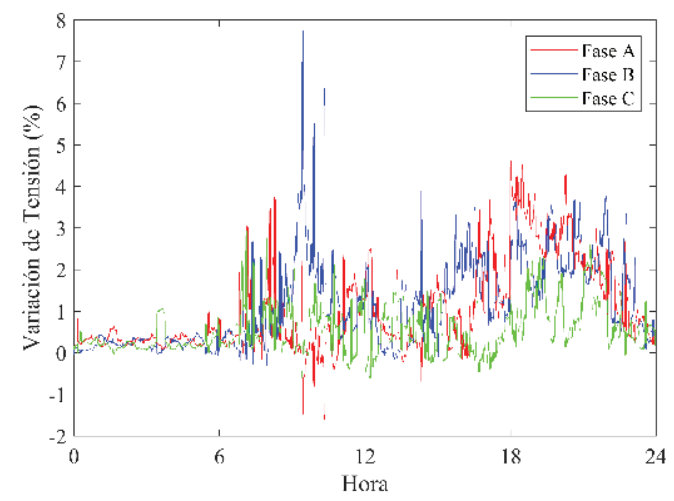

Figura 12. Caso II - Variación de tensión en la barra 906.

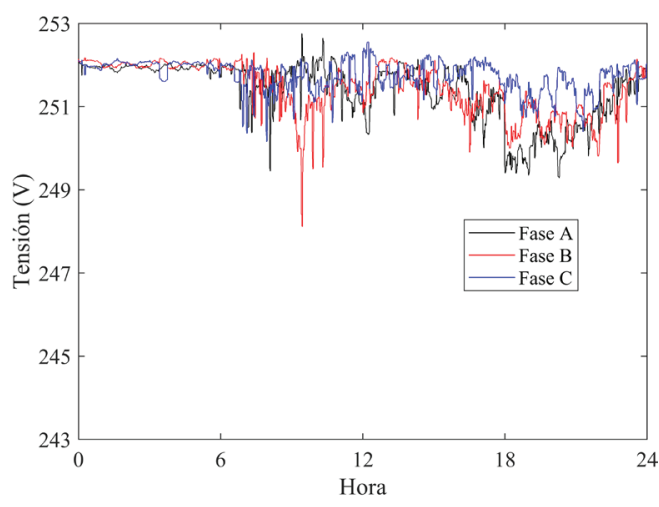

Figura 13. Caso II - Perfil de tensión en la barra 906.

Caso III:

Se analiza el nivel de incursión de VEHE en el año 2060 con incremento de hasta el 50\% en el parque automotor, adicionalmente se considera el incremento anual de 1\% hasta el 2060. La nueva demanda de energía se muestra en la figura 8. Para el incremento de demanda, las pérdidas también se incrementan, el pico máximo se presenta a las 18:00 horas (14 kW) según muestra la figura 14, en comparación con el caso base de la figura 5, representa un incremento de 11 veces las pedidas eléctricas.

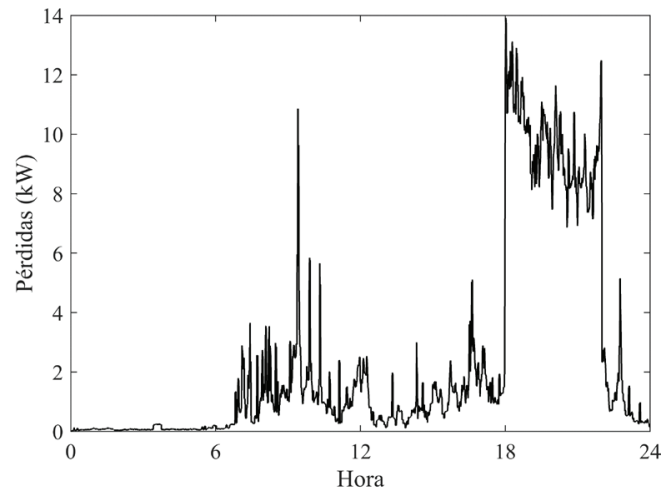

Figura 14. Caso III - Pérdidas totales en el sistema.

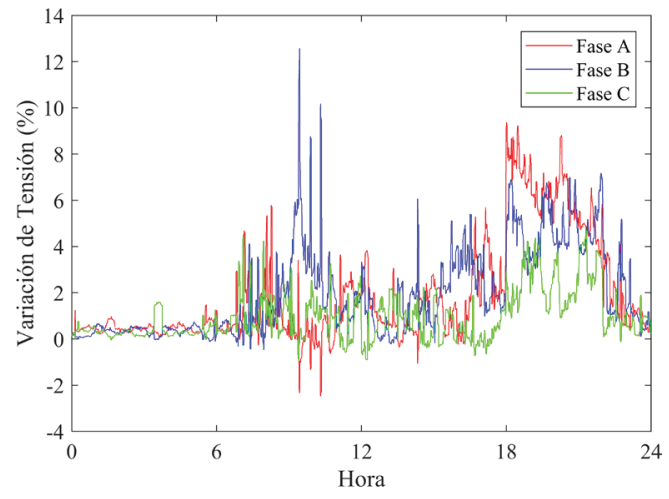

Figura 15. Caso III - Variación de tensión en la barra 906.

La figura 15 muestra la variación de tensión porcentual en la barra 906, a las 10:00 hora se registra la máxima variación con 12\%, en el horario de carga de VEHE se visualiza una variación de $8 \%$ y $6 \%$ en las fases $A$ y $B$ respectivamente. Estos valores superan a los límites establecidos en el NEC y el CNEP, Ios valores hallados, confirman la necesidad de repotenciación de la red de distribución para no alterar la calidad de suministro eléctrico para los usuarios finales y la operación estable del sistema eléctrico. 
Los valores nominales de la tensión en la barra 906 se muestran en la figura 16, en relación con la figura 15, los niveles de tensión disminuyen considerablemente en las tres fases durante el horario de conexión de VEHE.

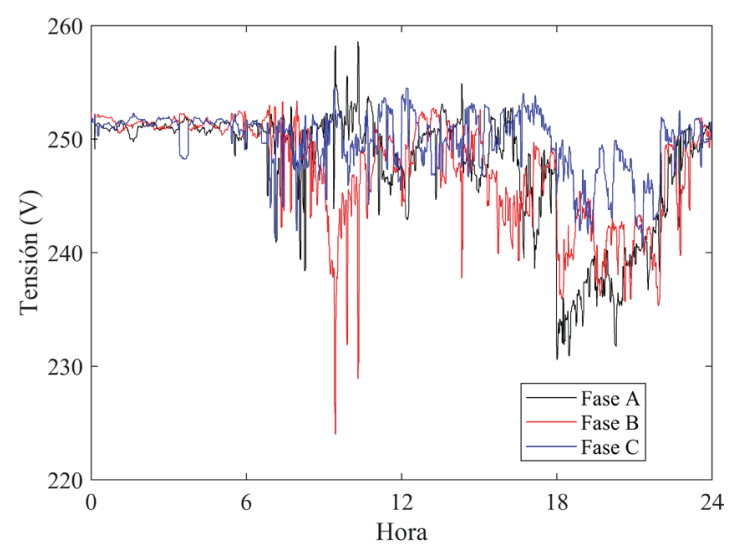

Figura 16. Caso III - Perfil de Tensión en la barra 906.

\section{Conclusiones}

Es importante señalar que la información utilizada en la simulación solo pertenece a la demanda registrada en 24 horas, para un análisis con mayor complejidad se podrá usar mayor cantidad de información. Respecto a la franja horaria donde se realiza la recarga, esta investigación considera que el consumo de energía de la batería es constante, en tanto para un análisis más realista podrá considerarse una curva de carga de un modelo matemático para la batería. Finalmente, el análisis realizado en los casos II y III representan el peor caso respecto al nivel de carga de batería, es decir la situación cuando el usuario agotó en su totalidad la capacidad de la batería, las personas llegan en simultaneo a sus hogares y la batería tiene un estado de carga prácticamente nulo.

En la presente investigación se analizó el comportamiento de la red de distribución eléctrica ante la incursión de vehículos eléctricos híbridos enchufables. En análisis considera el caso base sin VEHE, el caso con $35 \%$ y el caso con $50 \%$ de VEHE en el parque automotor. El incremento de la demanda eléctrica debido a esta nueva tecnología evidencia una mayor variación de tensión en las barras del sistema de distribución, los valores obtenidos superar al límite establecido por el Código Nacional de Electricidad Estadounidense y Peruano; las pérdidas también se incrementan, los resultados evidencias un incremento de más de 10 veces respecto al caso base ante una penetración de 50\% de VEHE. La evaluación de diferentes niveles de incursión de VEHE y el análisis de indicadores como la variación de tensión y las pérdidas eléctricas, evidencia la necesidad de repotenciar las redes de distribución y la instalación de equipos de regulación de tensión con el objetivo de preservar la calidad de energía eléctrica suministrada a los usuarios en sus domicilios y preservar la operación estable del sistema eléctrico.

\section{Agradecimientos}

Los autores agradecen a la Universidad Nacional del Centro del Perú (UNCP) y a la Facultad de Ingeniería Eléctrica y Electrónica por las facilidades financieras y técnicas para el desarrollo de la presente investigación. 


\section{Referencias}

[1] IRENA, "Electric vehicles: Technology brief," Abu Dhabi, 2017.

[2] H. Fathabadi, "Novel solar powered electric vehicle charging station with the capability of vehicle-to-grid," Sol. Energy, vol. 142, pp. 136-143, 2017.

[3] S. Shafiee, M. Fotuhi-Firuzabad, and M. Rastegar, "Investigating the impacts of plug-in hybrid electric vehicles on power distribution systems," IEEE Trans. Smart Grid, vol. 4, no. 3, pp. 1351-1360, 2013.

[4] M. Rastegar, M. Fotuhi-Firuzabad, and F. Aminifar, "Load commitment in a smart home," Appl. Energy, vol. 96, pp. 45-54, 2012.

[5] Y. Kongjeen and K. Bhumkittipich, "Impact of plug-in electric vehicles integrated into power distribution system based on voltage-dependent power flow analysis," Energies, vol. 11, no. 6, p. 1571, 2018.

[6] Z. Zhang and D. Gu, "Impacts of charging plug-in hybrid electric vehicles on the electric grid and its charging strategies," in 2012 Power Engineering and Automation Conference, 2012, pp. 1-4.

[7] M. D. Galus and G. Andersson, "Integration of plug-in hybrid electric vehicles into energy networks," in 2009 IEEE Bucharest PowerTech, 2009, pp. 1-8.

[8] W. Su, J. Wang, K. Zhang, and M.-Y. Chow, "Framework for investigating the impact of PHEV charging on power distribution system and transportation network," in IECON 2012-38th Annual Conference on IEEE Industrial Electronics Society, 2012, pp. 4735-4740.

[9] U.S. Energy Information Administration, "Annual Energy Outlook 2020," Washington, 2020.

[10] IEEE PES AMPS DSAS Test Feeder Working Group., "PES Test Feeder." [Online]. Available: https://site.ieee.org/ pes-testfeeders/. [Accessed: 10-Jan-2020].

[11] G. Dudgeon, "IEEE 906 Bus European LV Test Feeder in Simscape Power Systems." MATLAB Central File Exchange, 2018.

[12] M. Kintner-Meyer, K. Schneider, and R. Pratt, "Impacts assessment of plug-in hybrid vehicles on electric utilities and regional US power grids, Part 1: Technical analysis," Pacific Northwest Natl. Lab., vol. 1, pp. 1-20, 2007.

[13] MathWorks., "Solver." [Online]. Available: https://www.mathworks.com/help/simulink/gui/solver.html [Accessed: 10-Jan-2020]. 